\title{
Comprehensive Evaluation and Classification of Interchange Diagrammatic Guide Signs' Complexity
}

\author{
Yang Li, ${ }^{1,2}$ Xiaohua Zhao $\mathbb{D}^{1},{ }^{1}$ Qing He, ${ }^{2}$ Lihua Huang, ${ }^{1}$ and Jian Rong ${ }^{1}$ \\ ${ }^{1}$ College of Metropolitan Transportation, Beijing Key Laboratory of Traffic Engineering, Beijing University of Technology, \\ Chaoyang, China \\ ${ }^{2}$ Beijing Police College, Beijing, China
}

Correspondence should be addressed to Xiaohua Zhao; zhaoxiaohua@bjut.edu.cn

Received 1 January 2018; Accepted 19 March 2018; Published 23 April 2018

Academic Editor: Ángel Ibeas

Copyright (C) 2018 Yang Li et al. This is an open access article distributed under the Creative Commons Attribution License, which permits unrestricted use, distribution, and reproduction in any medium, provided the original work is properly cited.

\begin{abstract}
The effectiveness of interchange diagrammatic guide signs has significant meaning in traffic safety and driver's understanding. This paper presented a comprehensive evaluation and classification of interchange diagrammatic guide signs' complexity. The effectiveness of interchange diagrammatic guide signs relies on how well road users can understand those diagrams. This study tested 37 types of diagrams on the visual recognition complexity degree in three levels, general level, partial level, and detailed level, and finally seven indexes are selected to evaluation and classification of interchange diagrammatic guide signs' complexity. These indexes can be used to conduct quantitative evaluation and classification. And the result of diagram complexity range is between -1.366 and 2.046, which have a correlation with graph cognition complexity, including perspective of distribution, diagram character, essential element expression manner, and utilization degree, and $K$-means clustering method was used in the analysis. Based on the presented method, 37 types of diagrams are separated into three categories according to their complexity score: low complexity, medium complexity, and high complexity. This study not only presents a theoretical approach for quantitative evaluation of guide signs' complexity and effectiveness but also can be a reference for traffic sign design and application.
\end{abstract}

\section{Introduction}

Interchange diagrammatic guide signs are necessary traffic control device for urban traffic junctions. They are more intuitive, vivid, vibrant, and integrated in displaying and delivering information [1]. Unlike Europe and US whose guide signs are mainly arrows and simple graphics, guide signs with the interchange diagrams reflecting ramp placement as the main contents have been showing a rapid growth trend in China and other Asian countries (Figure 1). Take the city of Beijing as an example, 245 interchanges apply 369 diagrammatic guide signs in the urban area with a percentage of $75.3 \%$, according to one bridge with two signs. However, the effectiveness of interchange diagrammatic guide signs relies on road user's understanding of these diagrams [2]. The development process of interchange diagrams is from simple to complex. Corresponding to the complexity degree of interchange types and routes, the complexity of interchange diagrams also differs significantly. In fact, the complexity of interchange diagrams affects drivers' cognitive load and awareness level significantly. Experiences suggested that incredibly complex diagrams would confuse and horrify drivers, or even become a potential safety risk. Thus, it is of particular importance to apply research to the evaluation and classification method of graphics complexity.

\section{Literature Review}

Fontaine et al. [3] indicated that the United States is one of the first countries to conduct research on guide sign diagrams which started in the 1970s. The early research intended to study on contrasting advantages and disadvantages between graphical signs and conventional signs. With the increased popularization of guide sign diagrams, the research emphasis gradually turned to the improved design and efficient evaluation of sign graphics. More research results have appeared in the late 1990s and the beginning of this century. Chrysler et al. [4] pointed out the development variation in 

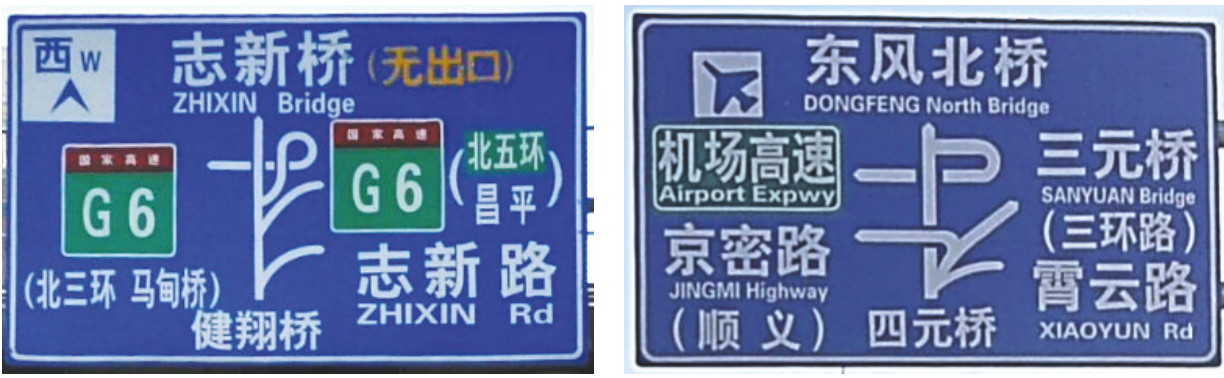

FIgure 1: Complex interchange diagrammatic guide signs.

their research report and summarized the study methods and results.

At present, the researches on guide sign diagrams still focus on analyzing the cognition effect in certain road conditions or the influence of some factors in the sign diagrams on cognition, which ignored the complexity of the diagrams themselves. Katz et al. [5] provided comparison result through contrasting five different settings including graph signs in exits of multilane interchanges with acceleration and deceleration lanes. Lin et al. [6] contrasted the recognition differences of graph signs under different typefaces and sizes, graphic patterns, and text-image system designing methods according to the graph characteristics in various countries. Di et al. [7] selected the time and visual accuracy of drivers searching sign information as an index and compared the differences between guide sign diagrams and cross diagrams by the one-way ANOVA.

For the complexity and classification of signs, recent researches mainly focus on the influences of information quantity, text size, and distribution of the text identification on drivers. The primary analysis objects are overall cognition performance and target searching performance. The main purpose is to confirm the threshold of sign information or compare the advantages and disadvantages of sign designing schemes. Cui [8] summarized the study status of guide sign text visibility and the amount of information and indicated that the existing researches all focused on the consciousness level and mainly studied the influences of physical parameters of text typeface and size and road names information on vision interpretation performance, which is not deeply into the understanding level of users understanding the route space. Li et al. [9] extracted and established evaluation index for accessing the effectiveness of traffic signs. Liu and Zhang [10] drew an analogy between road traffic sign information and generalized communication system with the quantitative evaluation method of road traffic signs and proposed the method for calculating entropy of signs according to the different levels of users understanding.

The diagrammatic interchange guide signs in Asian countries represented by China mostly use diagrams to describe ramp placements and travel routes. The diagrams have more forms and are more complicated. However, there was little research on the cognition of this kind of interchange signs. Enhancing the research and evaluation on the cognition characters of interchange guide sign diagrams could provide the basis for promoting the application of diagrammatic interchange guide signs and develop the related standards. Therefore, aiming at the issues of cognition complexity evaluation and classification of interchange diagrams, the compared data on the diagrams cognition covering three different levels (overall, partial, and detailed) were obtained through cognitive experiment. Furthermore, the method was proposed based on the comprehensive evaluation and classification of the cognitive complexity of the real 37 types of Chinese diagrams in Beijing by factor analysis and cluster analysis. The study results are useful for standardizing the use of different graphs, enhancing the comprehension and validity of complex guide signs, and improving the level of road safety.

\section{Methodology}

3.1. Design for Interchange Sign Diagrams. According to the design theory of interchanges, considering the factors such as ramp form, export arrangement, road crossing, urban road complex situation, and number of strokes, this study selects 37 existing Chinese interchange sign diagrams in Beijing, shown in Figure 2.

The cognition of interchange diagrammatic guide signs is closely related to the pattern of the graphic. This study selected diagrams from the perspective of design and the actual application, which could ensure the representativeness of study object. From drivers' cognition rules, studying the complexity degree and cognition characteristics of interchange diagrams could provide the foundation for the further design specifications.

3.2. Subjects. According to Central Limit Theorem, if a sum of random variables is normally distributed, a large sample size obtained from those variables also fits normal distribution. Besides, the sample size not less than 30 is a rule-of-thumb [11] and is commonly used in driving behavior empirical research. Besides, while it would have been desirable to have a higher number of subjects, it is common to obtain a small sample size, due to the high resource demands. For example, research into the nighttime legibility of traffic signs was performed with 24 participants [12]; research into the use of diagrammatic signs was conducted with 13 participants [13]; and a study aiming at the effects of eco-driving 


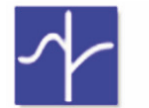

01.jpg

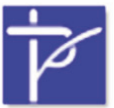

11.jpg

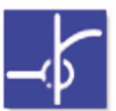

21.jpg

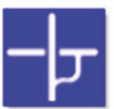

31.jpg

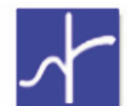

02.jpg

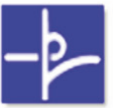

12.jpg

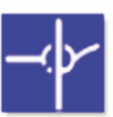

22.jpg

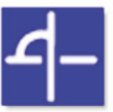

32.jpg

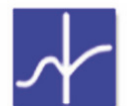

03.jpg

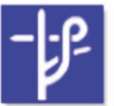

13.jpg

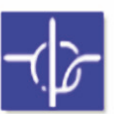

23.jpg

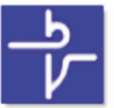

33.jpg

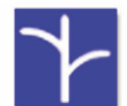

04.jpg

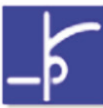

14.jpg

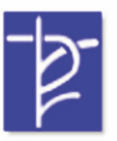

24.jpg

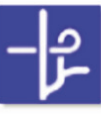

34.jpg

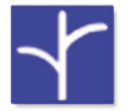

05.jpg

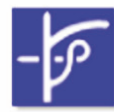

15.jpg

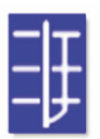

25.jpg

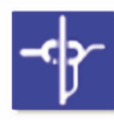

35.jpg

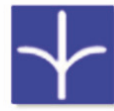

06.jpg

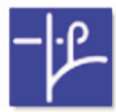

16.jpg

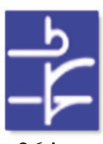

26.jpg

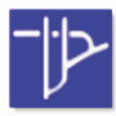

36.jpg
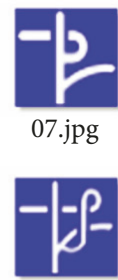

17.jpg

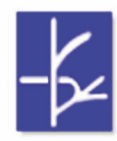

27.jpg

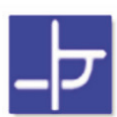

37.jpg

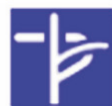

08.jpg

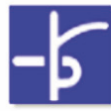

09.jpg
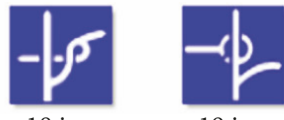

19.jpg

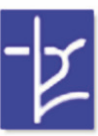

28.jpg

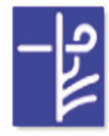

29.jpg

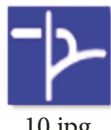

10.jpg

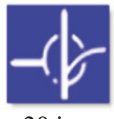

20.jpg

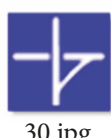

FIGURE 2: Interchange diagrams and number.
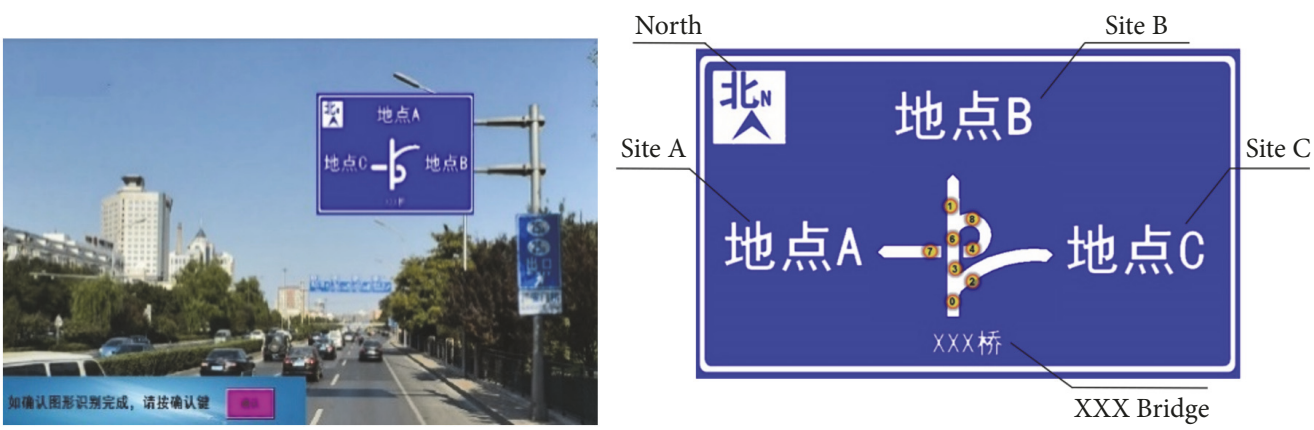

FIGURE 3: Program interface and diagrammatic guide signs pictures with the serial number.

training courses on driver behavior and comprehensibility was performed with 22 participants [14].

Thirty drivers with an average age of 35 were recruited. They were 24 males and 6 females and their ages ranged from 20 to 55. Each participant had a valid driver's license with 1 to 30 years driving experience, and the average was 12 years. All participants were healthy with no color weakness or color blindness and the UCVA (uncorrected visual acuity) was ensured in 0.5 or more.

3.3. Experiment Design. The experiment was controlled with a ThinkPad S3 notebook, and the test data were automatically recorded. From the driver's perspective, all the diagrams with the same background were randomly shown on the LCD with AOC55 inch monitor (screen resolution of 1080P, refresh rate of $85 \mathrm{~Hz}$ ). The distance between the subjects and the center of the screen is fixed at 2 meters, which was equivalent to a $50 \mathrm{~m}$ actual visual distance based on similar triangular scaling. Experimental picture effects are displayed in Figure 3.

During the experiment, 37 types of interchange diagrams were shown on the LCD randomly for avoiding the influences of the showing order on the results. The test of each diagram has consisted of 3 cognitive levels.
3.3.1. Complexity Test on Overall Recognition. It consisted of two parts, that is, confirmation of overall recognition and complexity evaluation.

Confirmation of Overall Recognition. Participants observed sign diagrams and pressed "Enter" key if they thought they had understood the meaning of the graph. For avoiding the influence of the specific site names, the destination name of each sign's image were expressed as "site A," "site B," "site C," and so forth. The operational time was limited to 30 seconds; any test over 30 seconds was considered as recognition failure.

Complexity Evaluation. Participants accessed the complex nature of the diagrams. Numbers 1-6 were on behalf of "very simple," "relatively simple," "simple," "difficult," "relatively difficult," and "very difficult." Participants were required to press the corresponding number keys for confirmation.

3.3.2. Complexity Test on Partial Recognition. For each diagram, the program randomly displayed a destination name on the screen, such as "Site A." Participants chose the exit to the destination and performed the driving operation with the operating selection key, including "Turn left," "Straight," 
and "Turn right," according to their understanding. Then the program would not stop to give the next destination randomly until all the corresponding destination export operation selection tests completed. Each destination was selected for a time limit of 10 seconds, and the locations of the destinations were randomly arranged to avoid repetitive effects.

3.3.3. Complexity Test on Detailed Recognition. It means the confirmation of driving route selection. Participants selected the number leading to the destination and filled the blanks, according to their understanding, and then they pressed "Enter" key to confirm. For example, in Figure 3, the number of "site A" was "036847."

The experiment included four parts for each participant.

(1) Demographic Questionnaires. Before driving, subjects were required to finish the questionnaires, which included subjects' basic information, physiological and psychological conditions at the pretest stage. For example, a driver should fill in forms about age, gender, driving experience, whether took drugs, tobaccos, whether drank alcoholic, tea, or caffeine drinks, whether had a regular circadian rhythm and slept disorderly, and so on. Drugs, tobaccos, and alcoholic or caffeine drinks were banned during the experiment. All of the drivers agreed and signed an informed consent before participating in the study.

(2) Subject Exercise. The program randomly provided two practice graphics for the subjects. Through the practice, the subjects could confirm the test requirements and become familiar with the overall graphics, local and detailed test of the process, and key operation method. At the same time, the practice program could test whether the subject adapted to the test equipment.

(3) Formal Test. Subjects were required to finish the task by themselves. Thirty-seven types of interchange diagrams were tested randomly, and each graph was tested in three terms in turn. Subjects' test data were recorded automatically by the program.

(4) A Subjective Questionnaire. Each participant tested 37 random diagrams in the formal test. After all tasks were completed, each participant was asked to finish a subjective questionnaire. Their answers were used to record and analyze their subjective perceptions about diagrams in the experiment.

\section{Result}

A total of 300 subjective questionnaires were issued. The results of the graphical recognition showed that over $80 \%$ of the participants recognized the interchange sign diagrams through the process from the overall recognition to the partial level and to the detailed level. In order to comprehensively access the recognition complexity of interchange diagrams, the data were analyzed from three aspects.

\subsection{Data Processing}

\subsubsection{Evaluation on Overall Recognition Complexity}

(1) Overall Recognition Time. Overall recognition time of diagrams is the time of drivers observing and understanding the complete graphs. It reflected the recognition and understanding of each graph as a whole. The one-way ANOVA ( $F$ $=2.283, p=0.004$ ) was conducted for the test result of 37 types of diagrams on overall recognition time. There were significant differences among average recognition time of 37 types of diagrams.

(2) Subjective Feeling of Complexity. The rating result of the diagrams complexity reflected the subjective evaluation of the diagrams complexity. One-way ANOVA $(F=10.03, p<$ 0.001 ) showed that there were significant differences between the average scores of 37 types of diagrams.

The analysis of subjective and objective data demonstrated that there were significant differences between the overall complexities of 37 types of diagrams. The recognition time showed that the average identification time of the most complex diagram (No. 23) was 1.94 times the easiest diagram (No. 3) with the difference value of $1.772 \mathrm{~s}$. The subjective scores showed that the average value of the hardest diagram (No. 25) was 2.71 times the easiest diagram (No. 1) with the difference value of 2 points.

Figure 4 describes that the subjective feelings of most diagrams coincided with or were close to the relative positions of the overall visual time, while some of the graphs were also biased. Therefore, the comprehensive evaluation must consider both factors, which were the subjective feeling and the overall recognition time.

4.1.2. Evaluation on Partial Recognition Complexity. During the driving process, the drivers concerned more on the information leading to the destination. Thus, the analysis selected the turning operation confirming time and error rate of each target to reflect the complexity of the pattern in the direction of the destination.

The destination of the sign was divided into three directions (left, straight, and right). If there were multiple exits in the same direction (such as No. 24-29), the average values would be taken as the exit data of that direction. The oneway ANOVA results indicated that there were significant differences between the 37 types of diagrams in the exits of left, straight, and right directions on the aspect of the turning operations average confirming time (as illustrated in Table 1).

In order to express the comprehension and difference of complexity of partial recognition, the average and the maximum value of indicators in the three directions were selected and depicted in Figure 5.

The experiment recorded 3,660 data sets of turning operational selections. The correct data were 3,477 with the accuracy of $95 \%$, which indicated that most drivers could correctly understand the exit portion of the diagrams and made the right turning decision. There were 183 error data sets, including 17 error records due to time-up. 
TABLE 1: The results of one-way ANOVA test on single direction's recognition time.

\begin{tabular}{lcccc}
\hline Direction & $F$ value & $p$ value & AV & SD \\
\hline Left & 3.218 & $<0.001$ & $2839.922 \mathrm{~ms}$ & 439.1919 \\
Straight & 4.082 & $<0.001$ & $2410.845 \mathrm{~ms}$ & 415.2805 \\
Right & 2.168 & $<0.001$ & $2501.569 \mathrm{~ms}$ & 295.2000 \\
\hline
\end{tabular}

Subjective data comparison of the overall recognition time of the 37 types of diagrams

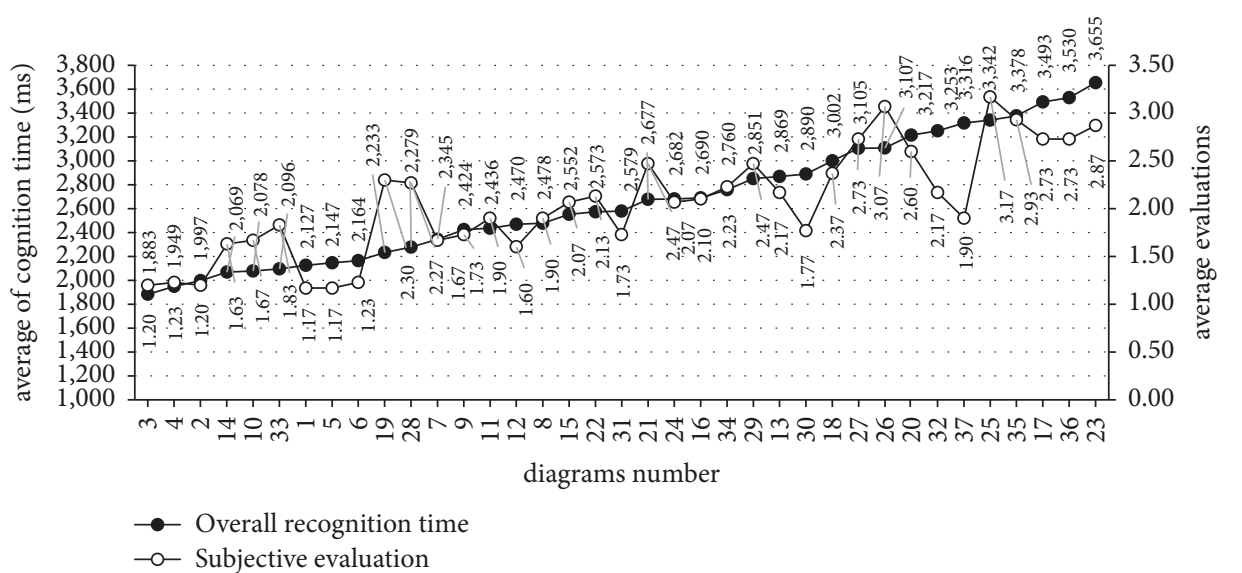

FIgURE 4: Subjective evaluation and visual recognition time.

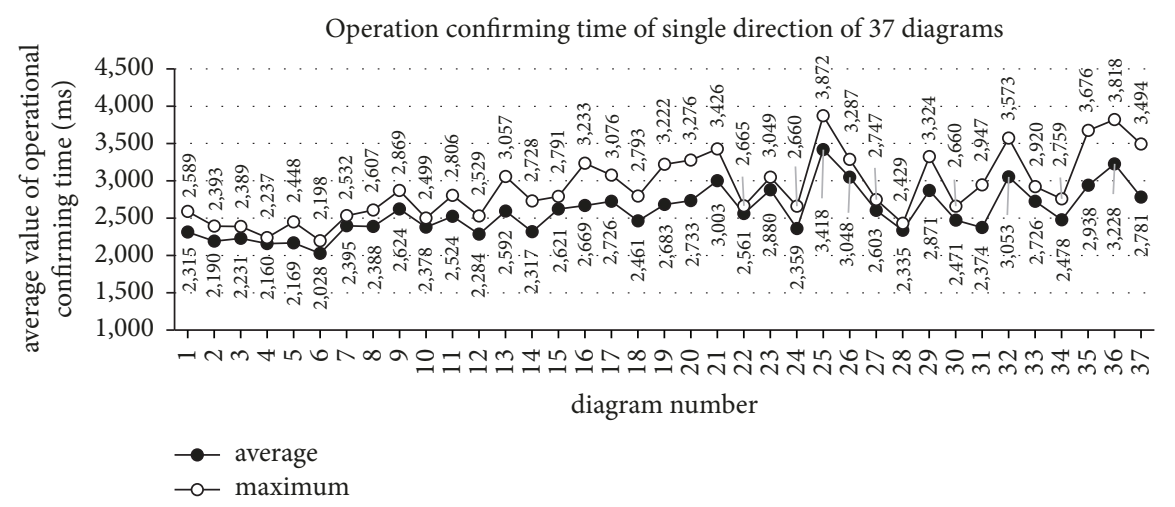

FIgURE 5: Single direction's recognition time.

Since the same diagram contained multiple destinations, each participant had multiple records in each diagram. Based on 37 types of diagrams, the number of errors and error ratios are shown in Figure 6.

The analysis results of partial recognition time and error rate data showed that there were significant differences in the partial complexity of 37 types of diagrams. The longest partial recognition time was $3,418 \mathrm{~ms}$ in No. 25, and the shortest was $2,028 \mathrm{~ms}$ in No. 6 , and the difference between these two values was $1,390 \mathrm{~ms}$. The partial recognition time was found to be consistent with the error rate on selecting operation, such as No. 9, No. 25, No. 32, and No. 36.

Scatter diagram was used to present the comparison result between overall recognition time and partial recognition time. Figure 7 showed that there were some negative correlations. The same diagram may have long partial recognition time and short overall recognition time (such as No. 33, No. 19, and No. 21) or short partial recognition time and long overall recognition time (such as No. 12 , No. 24, and No. 18). Therefore, in the design of diagrams, it is necessary to not only pay attention to the complexity of the partial graphics, but also concern about the combination of local graphics.

4.1.3. Evaluation on Detailed Recognition Complexity. In order to evaluate the detailed recognition complexity, each segment of the 37 types of diagrams was numbered. Participants selected the number according to their understanding to constitute integrated path sequence under the given destinations.

Analyzing the error data and easily mistaken point of selecting path sequence could reflect the detailed recognition 


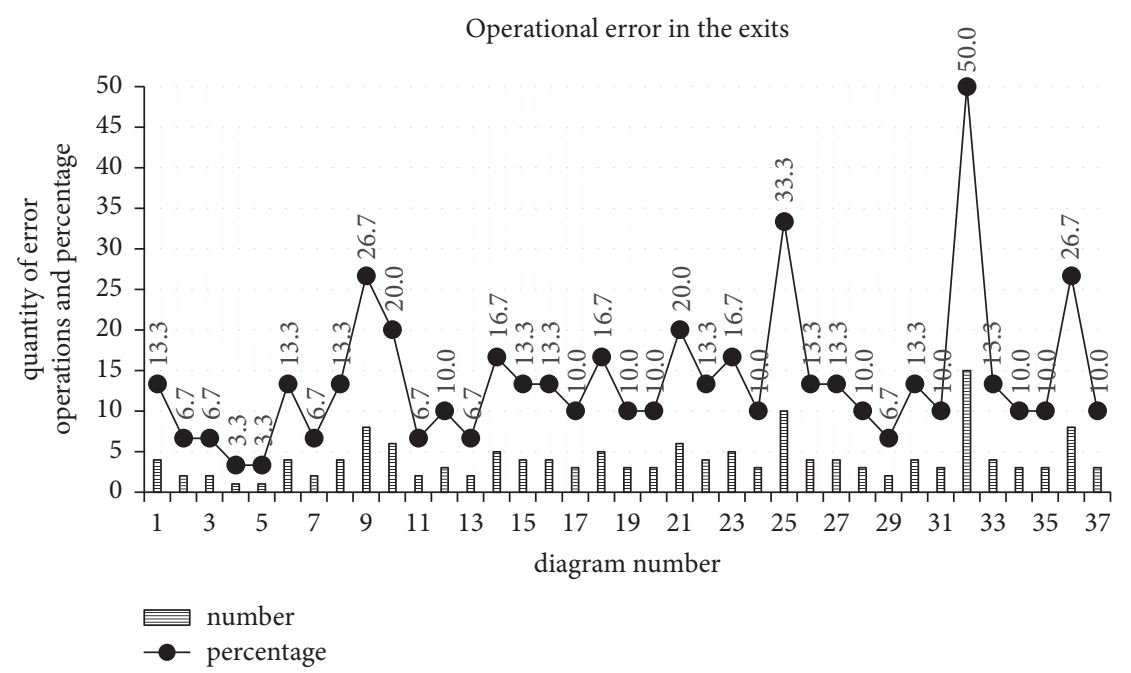

FIgURE 6: The number and ratio of exit operation error.

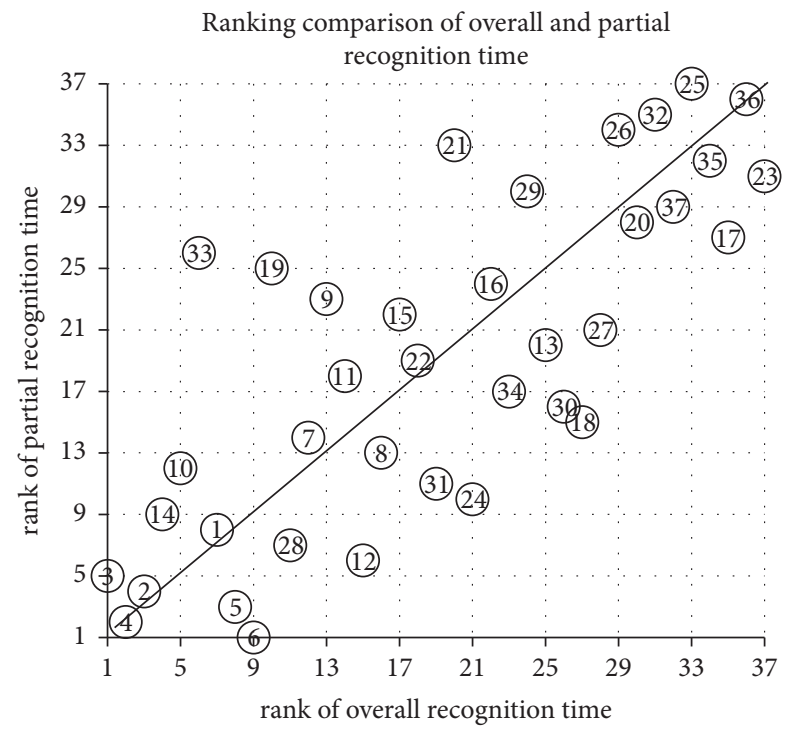

FIGURE 7: Ranking comparison.

complexity of the diagrams and also provide an essential basis for distinguishing the difficult position for comprehending.

The test recorded 3,660 path sequence selections. Through comparing with the right path, the coincident records were 3,488 with the accuracy of $95.3 \%$. It indicated that drivers could correctly understand most details of the graphs. In the 172 failure records, there were 49 records caused by impersonal factors, such as lack of noncritical number, poor eyesight, and incorrect operation on the keyboard. And the rest 123 records were caused by not understanding the meaning of the diagram or misunderstanding. According to the statistics of 37 types of diagrams, $70 \%$ diagrams were related to the detailed recognition error. Figure 8 showed the quantity and percentage of participants with wrong path sequences.
According to the content of sequence characters, the analysis compared each error record. In general, the primary cause of diagrams recognition error contained three aspects, the representation of ring ramp, the description of road crossing, and the description of the indirect left ramp.

The process of participants selecting path sequence was the recognition judging on the key points of the diagrams. The key points leading to wrong path selection were the risk points of detailed recognition on the diagrams. Table 2 showed the statistics of the risk points in 37 types of diagrams.

4.2. Comprehensive Evaluation. The data analysis indicated that there were certain relationships and negative correlations among the recognition complexity of the interchange sign diagram on the aspects of overall, partial, and detailed levels of recognition. For the comprehensive evaluation on the recognition complexity of interchange diagrams, seven variables in three dimensions were selected as the assessment indicators:

(V1) Overall recognition time

(V2) Average operational confirmation time in 3 directions (left turn, straight, and right turn)

(V3) Maximum operational confirmation time in 3 directions (left turn, straight, and right turn)

(V4) Proportion of participants who selected the wrong exit operation

(V5) Subjective assessment scores

(V6) Proportion of participants who chose the wrong path

(V7) Quantity of risk points

Due to the close relations among the seven variables, factor analysis was selected to evaluate comprehensively. The KMO test statistics of seven indicators was 0.745 which was above 0.5, and the Chi-square statistics of Bartlett's test was 


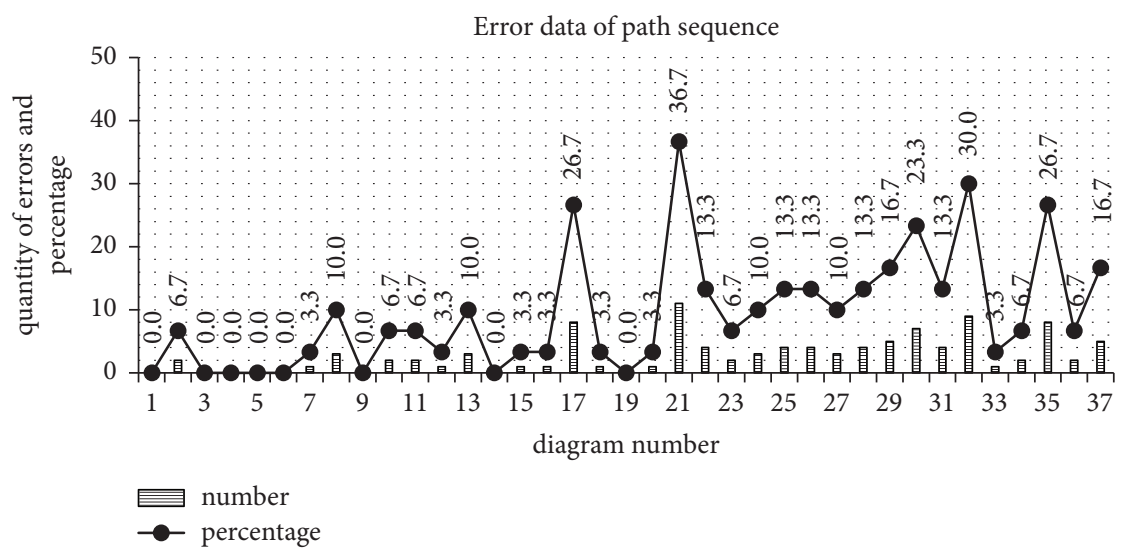

FIGURE 8: The number and ratio of path sequence error.

$215.579(p<0.001)$. The two statistics showed that factor analysis was suitable for evaluating on the recognition complexity of interchange sign diagrams. The factor analysis based on principal components is used to calculate the eigenvalue and contribution rate and obtains the total variance of the explanation. The analysis determined two factors $\left(F_{1}\right.$ and $\left.F_{2}\right)$, and the extracted information occupied $79.3 \%$ in the total of the initial data.

The loading formula of the common factors was calculated with regression algorithm:

$$
\begin{aligned}
F_{1}= & 0.195 x_{1}+0.205 x_{2}+0.197 x_{3}+0.119 x_{4} \\
& +0.195 x_{5}+0.156 x_{6}+0.157 x_{7} \\
F_{2}= & -0.064 x_{1}+0.233 x_{2}+0.225 x_{3}+0.659 x_{4} \\
& -0.082 x_{5}-0.342 x_{6}-0.565 x_{7} .
\end{aligned}
$$

It can be seen that the first principal component was related to the seven variables and the weight of each variable was almost equal to each other. The second principal component was more related to V4 and V7.

Based on formula (1), together with the proportion of the eigenvalues corresponding to the two common factors in the total extraction eigenvalues, the calculating method of comprehensive scores was obtained.

$$
Y=\frac{\left(F_{1} \times 64.835+F_{2} \times 14.491\right)}{79.326} .
$$

Table 3 listed the comprehensive scores and rank results of the 37 types of diagrams. The result indicated that there were differences between the entire evaluations of the recognition complexity of the 37 types of diagrams. The biggest difference in the complexity existed between No. 25 (score of 2.046, the highest) and No. 4 (score of -1.366 , the lowest) with the discrepancy of 3.412. The smallest difference in the complexity appeared between No. 24 and No. 8 with the variance of 0.004

Comprehensive evaluation results were to a certain extent in accord with the cognition rule, which reflected the objectivity and rationality of the method.
From the characteristics of the diagrams, in the diagrams with high recognition complexity scores, the proportion of diagrams was with more exports, more strokes, and intersections were high.

From the expression means of ramps, in the diagrams with low recognition complexity scores, the proportion of diagrams with directly turning left and directly turning right ramps was high.

From the forms of interchanges, in the diagrams with low recognition complexity scores, the typical interchange had high use ratio, and drivers were more familiar with them, such as No. 4, No. 12, No. 10, and No. 22.

Nevertheless, there was an inconsistency in the result of comprehensive evaluations. Some diagrams with simple strokes had the higher recognition complexity scores, such as No. 37 and No. 30. Some diagrams with more exits had the lower scores, such as No. 28 and No. 24.

4.3. Diagram Classification. According to the differences in diagram recognition complexity to determine the diagram classification, the interchange guide sign diagrams could be researched and used more appropriately. $K$-means algorithm was used to classify the 37 types of diagrams into three groups according to the seven indicators. Then, the grouping results were compared with the comprehensive rating rank result. Finally, the 37 diagrams were classified into three categories based on the low complexity, medium complexity, and high complexity, which was illustrated in Figure 9.

Based on the classification results, most of the diagrams which had low comprehensive evaluation scores with less recognition complexity were in group 1 . Most of the diagrams which had high overall assessment scores with greater recognition complexity were put in group 3 , and the diagrams with medium comprehensive evaluation scores were put in group 2.

The cluster analysis result was not entirely consistency with the comprehensive evaluation result. To classify the 37 types of diagrams according to the low, medium, and high complexity, the boundary diagrams were selected.

Figure 10 shows the differences of the graphs near the group's edges. The boundary between group 1 and group 2 


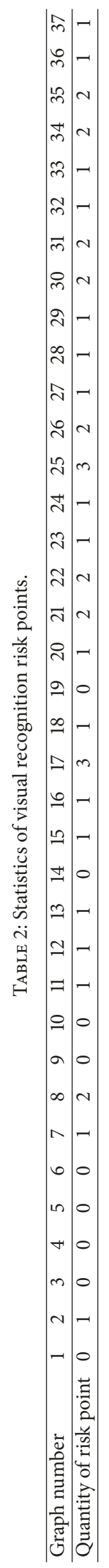


TABLE 3: Comprehensive evaluation.

\begin{tabular}{|c|c|c|c|}
\hline Total rank & Diagram number & Total score & Diagram \\
\hline 1 & 4 & -1.366 & \\
\hline 2 & 5 & -1.220 & \\
\hline 3 & 3 & -1.192 & \\
\hline 4 & 6 & -1.161 & \\
\hline 5 & 2 & -1.107 & \\
\hline 6 & 1 & -0.817 & \\
\hline 7 & 7 & -0.709 & \\
\hline 8 & 12 & -0.683 & \\
\hline 9 & 14 & -0.567 & \\
\hline 10 & 28 & -0.511 & \\
\hline 11 & 10 & -0.491 & \\
\hline 12 & 11 & -0.389 & \\
\hline 13 & 24 & -0.345 & \\
\hline 14 & 8 & -0.341 & \\
\hline 15 & 31 & -0.267 & \\
\hline 16 & 33 & -0.181 & \\
\hline 17 & 34 & -0.150 & \\
\hline 18 & 15 & -0.114 & \\
\hline 19 & 22 & -0.091 & \\
\hline 20 & 30 & -0.083 & \\
\hline 21 & 19 & -0.045 & \\
\hline 22 & 13 & -0.010 & \\
\hline 23 & 18 & 0.073 & \\
\hline 24 & 9 & 0.077 & \\
\hline 25 & 16 & 0.168 & \\
\hline 26 & 27 & 0.231 & \\
\hline
\end{tabular}

TABle 3: Continued.

\begin{tabular}{|c|c|c|c|}
\hline Total rank & Diagram number & Total score & Diagram \\
\hline 27 & 29 & 0.415 & 2 \\
\hline 28 & 20 & 0.431 & \\
\hline 29 & 37 & 0.506 & \\
\hline 30 & 17 & 0.672 & \\
\hline 31 & 23 & 0.811 & \\
\hline 32 & 26 & 0.914 & \\
\hline 33 & 21 & 0.997 & \\
\hline 34 & 35 & 1.075 & \\
\hline 35 & 36 & 1.569 & \\
\hline 36 & 32 & 1.854 & \\
\hline 37 & 25 & 2.046 & \\
\hline
\end{tabular}

was among the diagrams No. 14 , No. 28 , No. 10 , No. 11 , No. 24 , and No. 8. The biggest difference was 0.102 between diagram No. 10 and No. 11, which could be defined as the boundary of low complexity and medium complexity. With the same method, the biggest difference between group 2 and group 3 was 0.184 shown in the diagrams No. 27 and No. 29 which could be defined as the boundary of medium complexity and high complexity.

Thus, the classification results were as follows:

Low complexity diagrams: 4, 5, 3, 6, 2, 1, 7, 12, 14, 28, 10

Medium complexity diagrams: 11, 24, 8, 31, 33, 34, 15, $22,30,19,13,18,9,16,27$

High complexity diagrams: 29, 20, 37, 17, 23, 26, 21, 35, $36,32,25$.

\section{Conclusions}

This study provides a method for the complexity of interchange sign diagrams and four important contributions are listed as follows.

5.1. Recognition Differences. There are significant differences among the recognition performance of the 37 types of diagrams from the view of general, partial, and detailed.

5.2. Comprehensive Evaluation on Diagram Complexity. The comprehensive quantitative evaluation method on sign recognition complexity was proposed with factor analysis. 


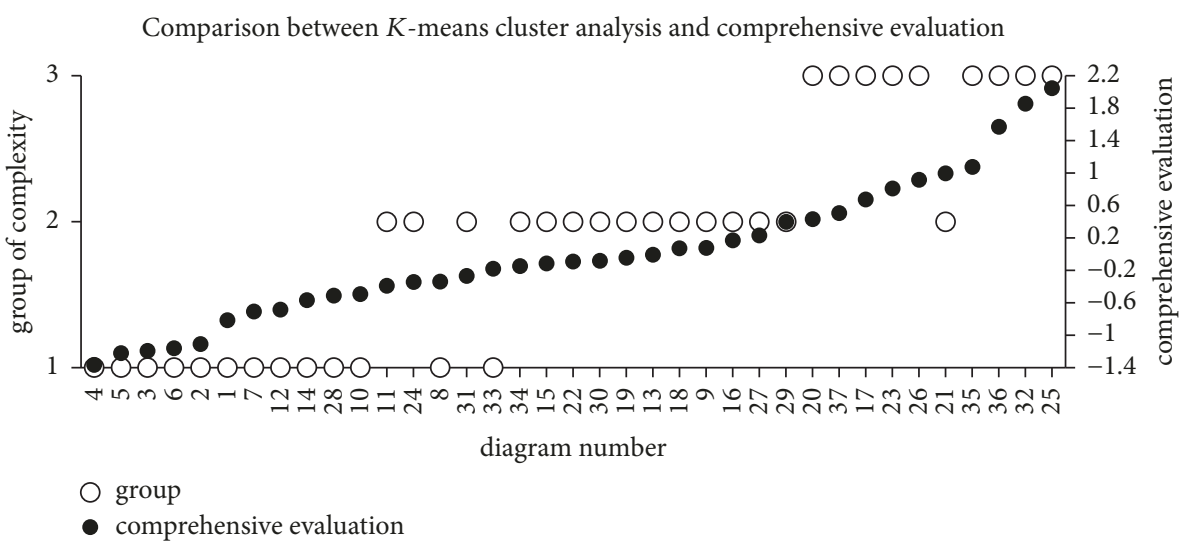

FIGURE 9: Comparison between classification and comprehensive evaluation.

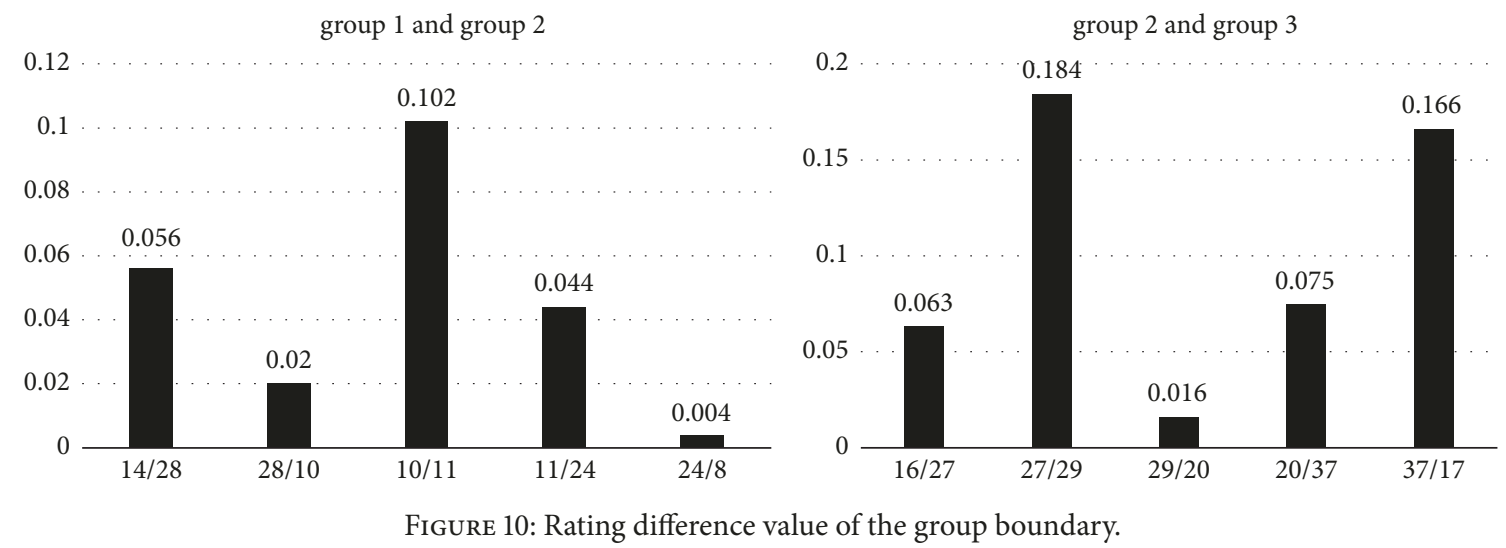

Thirty-seven diagrams were selected to verify the feasibility. The result shows that the recognition complexity of the 37 types of diagrams was among -1.366 and 2.046. Recognition complexity of interchange diagrams had a positive correlation with graph character, key element expression manner, and utilization degree.

5.3. Classification of Diagram Complexity. The $k$-mean algorithm was used to perform the cluster analysis on the 37 types of diagrams. The 37 diagrams were classified into three groups according to low complexity, medium complexity, and high complexity. The critical value was determined by comparing and ranking of comprehensive evaluation. Classification result is the basis of understanding and applying interchange guide sign diagrams and improving road safety.

\subsection{Comprehensive Evaluation and Classification Method.} All participants tested the whole process from general to partial and finally detailed level. Seven indicators measured diagram recognition complexity from these three levels. The comprehensive evaluation and classification method, based on factor analysis and cluster analysis, were to a certain extent in accord with the recognition rule, which reflected the objectivity and rationality of the method.

In conclusion, there were differences among the complexity of interchange guide sign diagrams. It will affect drivers' recognition of guide signs and is important for the research on the quantification and classification method of diagram's complexity. As real road conditions vary, 37 selected diagrams may not describe all the scenarios. To improve the applicability of the method, more interchange diagrams of other countries and areas could be analyzed to improve the findings and results further.

\section{Discussion}

The complexity of interchange diagrams affects drivers' cognitive load and awareness level significantly. This study is aimed at quantifying and classifying the complex degree of recognition of interchange diagrams, providing a reference for the design and simplification of complex signs, and providing the theoretical basis for deploying the guiding signs.

As mentioned above, there were 30 subjects participating in this experiment, so as to evaluate the effectiveness of 37 types of diagrams on interchange diagrammatic guide signs. Although subjects with the similar amount as this research were recruited in other driving behavior experiments, a small size would limit research results to some degree, and much more participants from various demographic groups could contribute to improve the results or conclusions. Therefore, a larger sample size will be chosen in future research to further 
explore the effects of interchange diagrammatic guide signs on driving behavior and comprehensibility.

The desktop cognitive experiment was designed in this study. Although some measures have been taken to ensure the accuracy of the data from the experimental picture display, experimental process control, and experimental data record, there are still some absolute errors which inevitably exist to some degree. However, from the perspective of the research objectives, which is to consider the difference between the visual recognition of interchange diagrams in terms of relative accuracy, the experimental data can support the analysis of the results. In the follow-up study, dynamic simulation experiment will be used to realize the excavation of the law in the course of driving. It will correct and perfect the deficiency of static experiment.

It is also important to analyze the driver's gaze data. The analysis helps to study the characteristics of the driver in terms of the details of the graphics and then to support the evaluation of graphics complexity and classification results. All subjects wore the eye tracker in this test. Further research could concentrate more on the trajectory of the fixation points.

According to the study results, there were obvious differences in the complexity of the diagrams, and the recognition complexity of some signs was very high, which will be beneficial in actual work. From the driver characteristics, in terms of visual distance and response time fixed, it was difficult for the drivers to complete the recognition of complex graphic signs and make the correct judgments. Therefore, for highly complex signs, in order to improve the setting effect, it is likely to take the split, set ahead, repeat the settings, and deploy auxiliary marking and other measures.

\section{Conflicts of Interest}

The authors declare that there are no conflicts of interest regarding the publication of this paper.

\section{Acknowledgments}

This study is supported by the BMCT (Beijing Municipal Commission of Transport) Project and the National Key Technology Support Program: Research on Effectiveness Evaluation and Optimization Method of Installation of Interchange Exit Guide Signs in Urban Expressway (2016-kjc-01-002); Key Technology and Status Research and Development of Driving Conditions on Freeways-Control Strategy Research in Work Zone (2014BAG01B01).

\section{References}

[1] C. H. Lin, K. C. Lin, C. Y. Leung, and etal., "Graphic recognition on highway diagrammatic guide sign," in Proceeding of the 5th International Congress of International Association of Societies of Design Research, Tokyo, Japan, 2013.

[2] X.-H. Zhao, L.-H. Huang, and J. Rong, "Influence of the interchange pattern guide sign on running speed in an urban complex interchange," Beijing Gongye Daxue Xuebao/Journal of
Beijing University of Technology, vol. 41, no. 9, pp. 1405-1414, 2015.

[3] M. D. Fontaine, S. T. Chrysler, and G. L. Ford, "Freeway guide signing: review of past research: final report," FHWA/TX-02/04170-1, Texas Transportation Institute, April 2002.

[4] S. T. Chrysler, A. A. Williams, D. S. Funkhouser, A. J. Holick, and M. A. Brewer, "Driver comprehension of diagrammatic freeway: Final report," Research Report 0-5147-1, Texas Transportation Institute, October 2006.

[5] B. J. Katz, E. E. Dagnall, M. A. Bertola, C. C. O'Donnell, and J. Shurbutt, "Evaluation of truncated arrow per lane guide signs," Journal of the Transportation Research Board, vol. 2434, pp. 8994, 2014.

[6] C. H. Lin, K. C. Lin., C. Y. Leung et al., "Graphic recognition on highway diagrammatic guide sign," in Proceeding of the 5th International Congress of International Association of Societies of Design Research, Tokyo, Japan, 2013.

[7] S. D. Di, M. Jiang, C. W. Jiao, and etal., "a study on maximum quantity of information for different kind of direction signs based on information searching time," Highway, vol. 7, pp. 255258, 2011.

[8] Z. H. Cui, The influence of text layout and amount of information of urban guide sign on road network space representation, Zhejiang Sci-Tech University, 2015.

[9] Y. L. Li, P. Cao, and Q. Dai, "Study on the effectiveness evaluation of traffic signs based on ergonomics," Journal of Highway and Transportation Research and Development, vol. 5, pp. 225229, 2009.

[10] X. Liu and K. Zhang, "Quantitative Evaluation Method of road traffic signs," Chinese Journal of Ergonomics, vol. 4, pp. 23-26, 2003.

[11] Z. N. Li and W. Q. Pan, Econometrics, Higher Education Press, Beijing, China, 3rd edition, 2010.

[12] T. Susan, J. Chrysler Paul, and H. Gene Hawkins, "Nighttime legibility of traffic signs as a function of font, color, and retroreflective sheeting," in Transportation Research Board 82th Annual Meeting, Wash, USA, 2003.

[13] H. T. Zwahlen, A. Russ, J. M. Roth, and T. Schnell, "Effectiveness of ground-mounted diagrammatic advance guide signs for freeway entrance ramps," Journal of the Transportation Research Board, vol. 1843, no. 1, pp. 70-80, 2003.

[14] Y. Wu, X. Zhao, J. Rong, and Y. Zhang, "How eco-driving training course influences driver behavior and comprehensibility: a driving simulator study," Cognition, Technology \& Work, vol. 19, no. 4, pp. 731-742, 2017. 


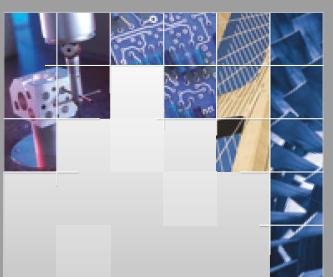

\section{Enfincering}
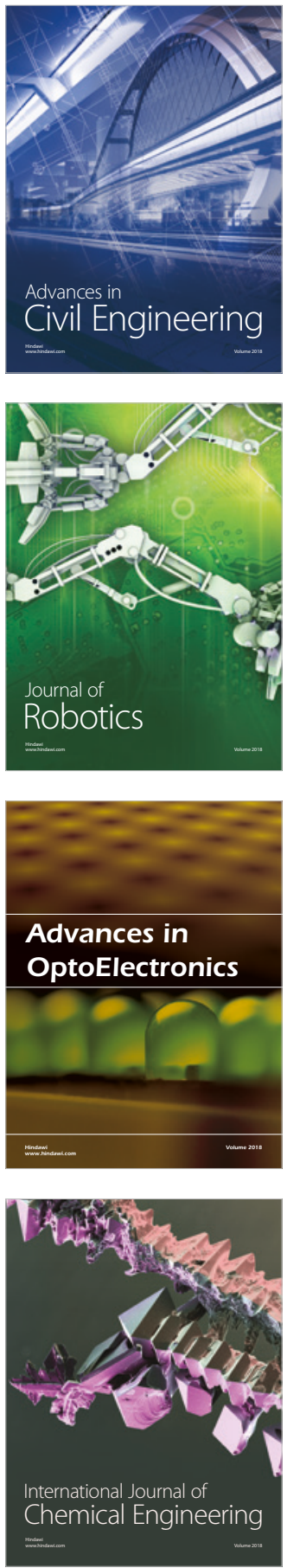

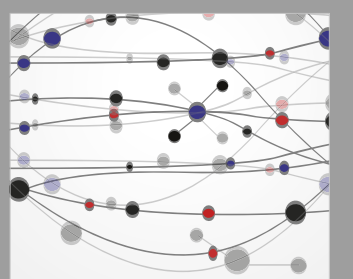

\section{Rotating \\ Machinery}

The Scientific World Journal

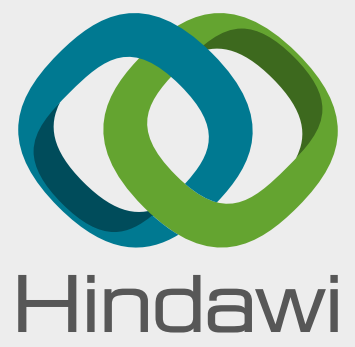

Submit your manuscripts at

www.hindawi.com
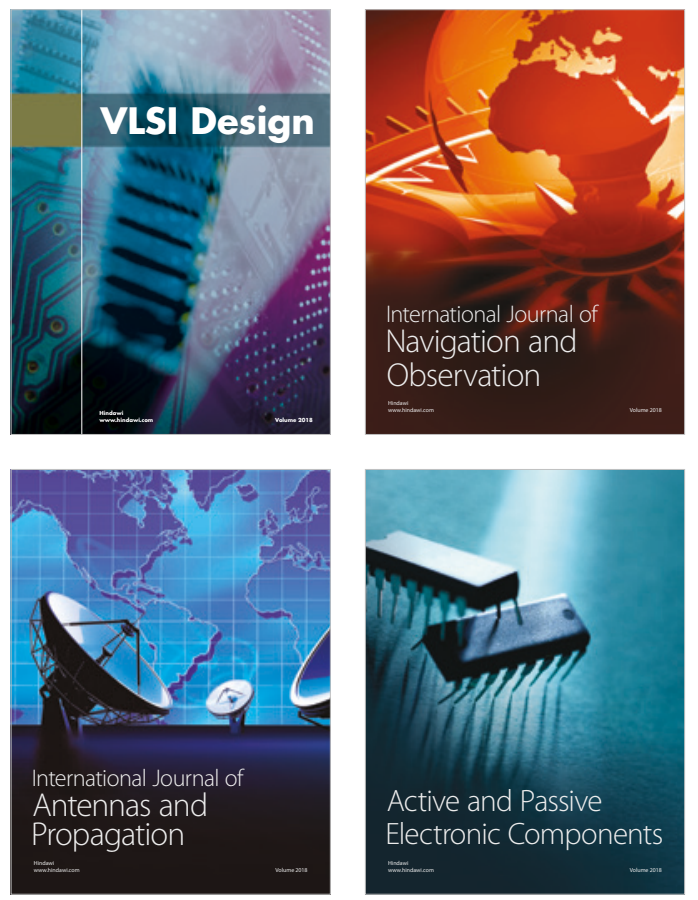
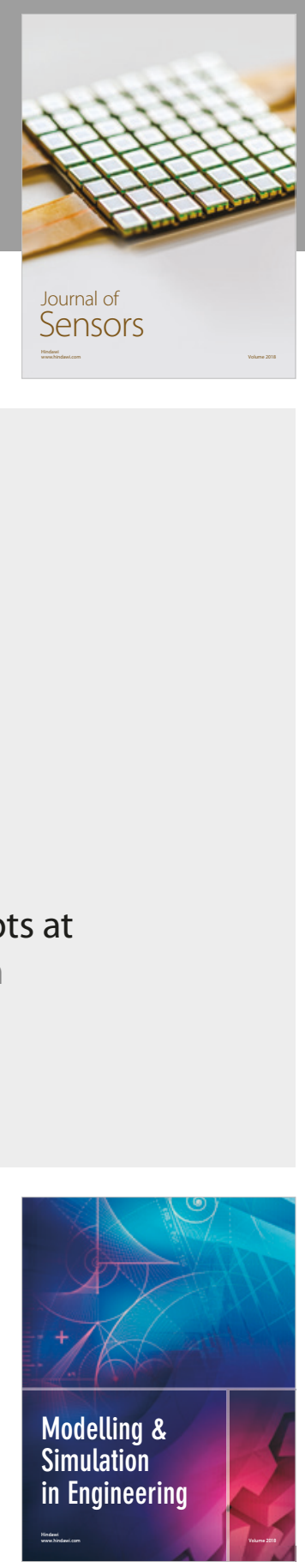

\section{Advances \\ Multimedia}
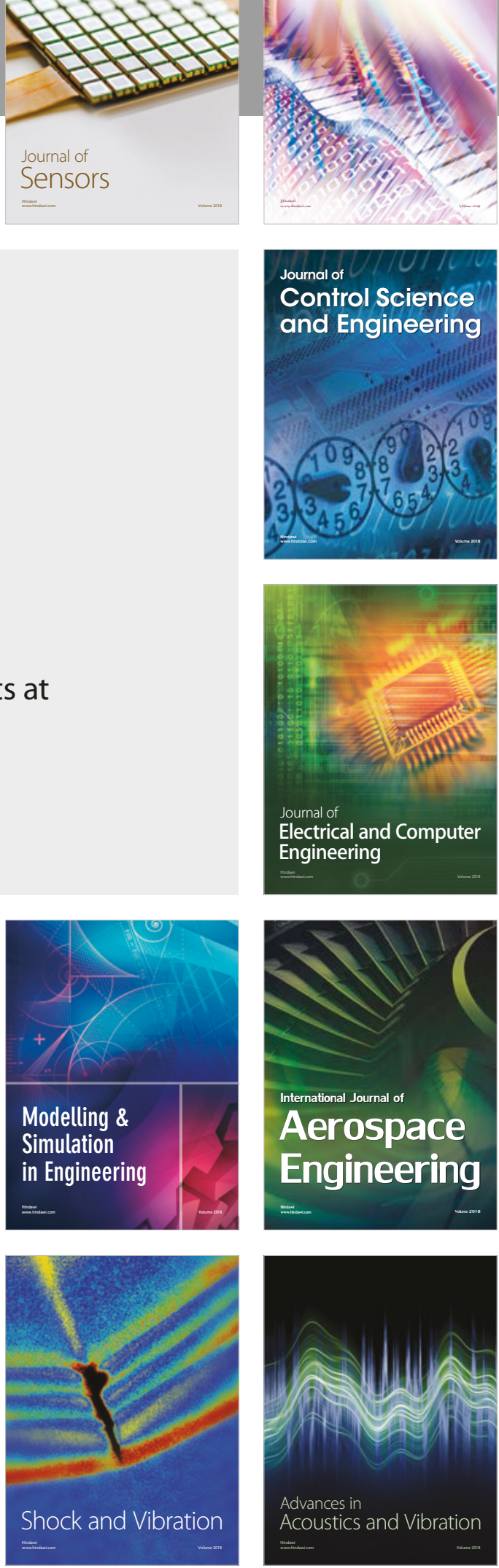